\title{
Descrição da avaliação de força muscular em idosas praticantes de atividades físicas
}

\author{
Joceléia Müller Ponte", Renan Donati**, Hugo Tourinho Filho***, Adriano Pasqualotti ${ }^{* * * *}$
}

\section{Resumo}

Com o envelhecimento humano, ocorrem muitas perdas, em especial as funcionais e musculoesqueléticas. O principal objetivo desta pesquisa quantitativa de caráter descritivo foi medir o pico de torque muscular dos membros inferiores e superiores, nos movimentos de flexão e extensão, bilateralmente, em 14 mulheres idosas com média de idade de 71,7 anos, todas praticantes de atividades físicas em um clube recreativo do município de Passo Fundo RS. A avaliação da força muscular foi realizada com o dinamômetro isocinético com-

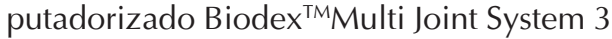
Pró $^{\circledR}$. O teste foi realizado bilateralmente nos movimentos de extensão/flexão de joelho nas velocidades angulares de $60 \% \mathrm{~s}$ e $180 \%$ s; para ombro, utilizou-se a velocidade angular de $60 \%$ s e $120 \%$ s. Foi possível verificar que o pico de torque é maior no joelho esquerdo e no movimento de extensão, na velocidade angular de 60\% $\%$. Em membro superior, nas velocidades angulares de $60 \%$ s e $120 \%$ s, destaca-se o movimento de extensão. Os resultados da pes- quisa indicaram que os membros inferiores foram mais influenciados pela prática da atividade física que os membros superiores. Nesse sentido, conclui-se pela importância de um programa de treinamento de exercícios físicos, no intuito de melhorar a aptidão física e, assim, prolongar a autonomia funcional ao longo da vida.

Palavras-chave: Envelhecimento. Dinamômetro de força muscular. Joelho. Ombros.

\section{Introdução}

O envelhecimento é um processo natural da vida humana e apresenta uma série de transformações biopsicossociais que alteram a relação do homem com o meio no qual está inserido. (ULBRICHT; CASSOL, 2005). Nesse contexto, a fisiologia do envelhecimento mostra que a capacidade humana de gerar força

* Fisioterapeuta. Mestranda do Programa de Pós-Pós-Graduação em Envelhecimento Humano da Universidade de Passo Fundo. Endereço para correspondência: Rua Carlos França, 2940, Centro, CEP: 98855-000, Entre-Ijuís - RS. E-mail: joce_ponte@hotmail.com.

*** Discente do curso de Educação Física da Universidade de Passo Fundo. Bolsista Probic/Fapergs.

**** Educador Físico. Doutor em Educação Física pela Universidade de São Paulo. Docente da Escola de Educação Física e Esporte da Universidade de São Paulo.

**** Matemático. Doutor em Informática na Educação e mestre em Ciência da Computação pela Universidade Federal do Rio Grande do Sul. Docente do Programa de Pós-Graduação em Envelhecimento Humano da Universidade de Passo Fundo.

$\hookrightarrow$ Recebido em setembro de 2012 - Aprovado em outubro de 2012.

$\rightarrow$ doi:10.5335/rbceh.2012.034 
muscular diminui com a idade, especialmente após os 60 anos. (ERNESTRO et al., 2009).

Com o envelhecimento, ocorrem perdas de massa musculoesquelética, as quais estão entre os problemas de saúde mais prevalentes e sintomáticos da meia-idade e da idade senil. (PRENTICE; VOIGHT, 2007). Esses mesmos autores ressaltam que a força é uma das mais importantes valências físicas e que a fraqueza muscular pode levar a pessoa idosa à incapacidade funcional, impedindo-a de realizar as atividades comuns da vida diária, tais como levantar-se de uma cadeira, tomar banho, vestir-se e até mesmo alimentar-se sozinha, além de tarefas domésticas como varrer o chão, ou colocar o lixo na rua. (TARTARUGA et al., 2005).

A perda de força muscular dos sujeitos está claramente relacionada com os níveis de atividade física praticada. Assim, aqueles que se mantêm ativos reduzem de forma considerável o declínio de força, prorrogando os efeitos do envelhecimento sobre o corpo humano. (PRENTICE; VOIGHT, 2007).

Nessa perspectiva, a prática de exercícios físicos para os idosos resulta em ações benéficas. Independentemente de sua origem ou natureza, qualquer tipo de atividade física favorece o idoso nos aspectos físicos, sociais e psicológicos, contribuindo para mantê-lo saudável. (BARBOSA, 2007).

O dinamômetro isocinético é um instrumento usado para avaliar a for- ça muscular desejável que possibilita a geração de dados quantitativos com objetivos válidos e confiáveis. (SONZA; ANDRADE, 2012). Destaca-se por ser capaz de coletar informações sobre as propriedades neuromusculares durante a contração dinâmica e com velocidade constante do músculo avaliado. (DVIR, 2002).

As atividades físicas devem ser incentivadas a desenvolver força muscular necessária para a realização de tarefas funcionais. Sendo assim, surge a necessidade de constatar quais são os grupos musculares mais suscetíveis às lesões, quais devem ser melhorados, além de procurar identificar e avaliar as possíveis fraquezas musculares. $\mathrm{O}$ objetivo do estudo foi avaliar o pico de torque muscular dos membros inferiores e superiores, nos movimentos de flexão e extensão, bilateralmente, e comparar a força dos músculos de joelho, ombro direito e esquerdo em idosas praticantes de atividade físicas.

\section{Material e métodos}

Estudo quantitativo de caráter descritivo. A amostra constou de 14 mulheres idosas que praticam atividades físicas em um clube recreativo do município de Passo Fundo - RS.

A amostra foi não probabilística, composta por idosas que expressaram interesse em participar da pesquisa. Todas foram informadas sobre os riscos e benefícios do projeto, aprovado pelo Comitê de Ética em Pesquisa da Universidade 
de Passo Fundo (Parecer 529/2011), e assinaram o Termo de Consentimento Livre e Esclarecido.

Com relação aos critérios de inclusão, as mulheres deveriam ter 60 anos ou mais e ser praticantes de programas de atividade física por no mínimo dois meses. Constituiu-se como critério de exclusão a presença de patologias que pudessem afetar a força muscular ou capacidade funcional de membros superiores e inferiores, impedindo que se realizassem os testes e as avaliações, ou de problemas cardíacos e/ou musculoesqueléticos que pudessem comprometer a integridade física da idosa.

Aplicou-se uma ficha de anamnese que constava de questões clínicas, laboratoriais e sociodemográficas, tais como escolaridade, medidas antropométricas (peso e altura), existência de alguma doença diagnosticada, frequência ao médico, uso de medicamento e atividades físicas. Em seguida, realizou-se a avaliação do isocinético, seguindo as orientações do fabricante.

As avaliações da força muscular foram realizadas no Laboratório de Biomecânica da Faculdade de Educação Física e Fisioterapia da Universidade de Passo Fundo. A Figura 1 apresenta uma imagem do dinamômetro isocinético computadorizado Biodex ${ }^{\mathrm{TM} M u l t i}$ Joint System 3 Pró ${ }^{\circledR}$ (BIODEX, 2002), equipamento utilizado nessas avaliações.

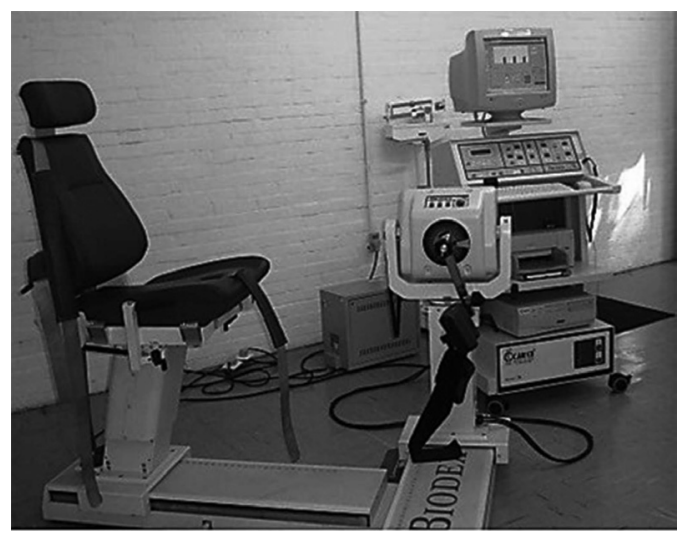

Figura 1 - Dinamômetro isocinético computadorizado Biodex ${ }^{\mathrm{TM}}$ Multi Joint System 3 Pro ${ }^{\circledR}$.

Inicialmente, foram realizados aquecimento de 5 min em bicicleta ergométrica sem carga e alongamento dos membros inferiores. Após, cada idosa, individualmente, foi posicionada no dinamômetro isocinético computadorizado, com ajustes de $90^{\circ}$ no assento e inclinação de $85^{\circ}$ no encosto, sendo estabilizada com os cintos de ombro, cintura e coxas. Assim, o eixo de rotação do dinamômetro foi alinhado com o eixo da articulação do joelho. O teste foi realizado bilateralmente nos movimentos de extensão/flexão de joelho nas velocidades angulares de $60 \%$ s e $180 \%$ s por cinco repetições cada e um intervalo de $30 \mathrm{~s}$ entre as séries.

Para a avaliação do membro superior - ombro -, a idosa realizou um aquecimento com movimentos ativos de flexão e extensão de ombro e, após o alongamento, procedeu-se a novo ajuste do assento em $15^{\circ}$ e inclinação do encosto em 70 a $85^{\circ}$. O indivíduo foi estabilizado com os cintos de ombro e cintura. O eixo 
de rotação de compromisso escolhido foi o processo acromial no plano sagital. Para a mensuração dos movimentos de flexão/ extensão do ombro, foram utilizadas as velocidades angulares de $60 \%$ s e $120 \% \mathrm{~s}$ por cinco repetições cada e um intervalo de $30 \mathrm{~s}$ entre as séries. Em ambas as avaliações, houve comandos verbais estimulantes com intensidades moderadas.

Para a estruturação do banco de dados, utilizou-se o aplicativo Epi Info $^{\mathrm{TM}}$ 3.5.1, e para a descrição dos resultados, o programa estatístico R i386 2.15.1. Para descrever as características sociodemográficas e a força muscular da amostra, empregaram-se as frequências relativas e as medidas de estatística descritiva.

O estudo compreende algumas limitações: a) as restrições musculoesqueléticas apresentadas pelas idosas na realização do dinamômetro isocinético, principalmente em membros superiores; b) a presença de sinais e sintomas de patologias osteoarticulares em joelho e ombro; c) a diminuição da amplitude de movimento articular decorrente da idade.

\section{Resultados e discussões}

A amostra foi constituída por 14 idosas, cuja média de idade foi de 71,7 anos, com um desvio padrão de 7,7 anos. A Tabela 1 descreve as características sociodemográficas (faixa etária e escolaridade), os fatores de risco (medicamentos de uso contínuo) e as morbidades (diabetes mellitus, anemia, doença respiratória aguda, doença vascular periférica) dos indivíduos que participaram da pesquisa.

Tabela 1 - Características sociodemográficas, fatores de riscos e morbidade dos indivíduos da amostra. Passo Fundo, 2012.

\begin{tabular}{llcc}
\hline Variáveis & \multicolumn{1}{c}{ Categorias } & $\mathrm{n}$ & $\%$ \\
\hline \multirow{2}{*}{ Faixa etária } & Menos de 75 anos & 8 & 57,1 \\
& 75 anos ou mais & 6 & 42,9 \\
Escolaridade & Ensino fundamental & 7 & 50,0 \\
Medicamentos de uso & Ensino médio & 5 & 35,7 \\
contínuo & Ensino superior & 2 & 14,3 \\
Diabetes mellitus & Sim & 13 & 92,9 \\
& Não & 1 & 7,1 \\
Anemia & Sim & 2 & 14,3 \\
& Não & 12 & 85,7 \\
Doença respiratória aguda & Sim & 2 & 14,3 \\
& Não & 12 & 85,7 \\
Doença vascular periférica & Não & 2 & 14,3 \\
& Sim & 12 & 85,7 \\
\hline
\end{tabular}


De acordo com os resultados descritos na Tabela 1 , há 8 mulheres com menos de 75 anos, o que corresponde a $57,1 \%$ da amostra. Desta, $50 \%$ têm até o ensino fundamental. Com relação aos fatores de riscos, 13 mulheres, $92,9 \%$ da amostra, usam medicamentos de forma contínua. No que se refere às morbidades, $85,7 \%$ não apresentam prevalência de diabetes mellitus, anemia ou doença respiratória aguda; por sua vez, $4 \mathrm{mu}$ lheres, $28,6 \%$, sofrem de doença vascular periférica.

Acerca da quantidade de medicamentos utilizados diariamente, os resultados evidenciam uma variação entre $0 \mathrm{e}$ 8 , com uma média de 2,9 medicamentos e um desvio padrão de 1,9 medicamentos. Quanto ao tempo a que participam das atividades físicas, os resultados mostram um período que varia entre dois meses e mais de oito anos. Já no que diz respeito à frequência com que participam das atividades físicas, os resultados variam entre duas e cinco vezes por semana.

Em relação ao índice de massa corporal (IMC), verifica-se uma média de $28,6 \mathrm{~kg} / \mathrm{m}^{2}$, com um desvio padrão de 4,1 $\mathrm{kg} / \mathrm{m}^{2}$. Segundo tabela da Organização Mundial em Saúde (WHO, 1997) para idosos, que é diferente da tabela IMC para adultos, esses valores indicam que as participantes estão levemente acima do peso.

Os problemas crônicos tornam-se mais frequentes com a idade, acumulando-se conforme a vida dos indivíduos se prolonga, e estima-se que $80 \%$ dos idosos com 65 anos ou mais apresentam uma ou mais doenças crônicas. (PREN-
TICE; VOIGHT, 2007). Na Tabela 2, apresentam-se as médias e o desvio padrão dos resultados do pico de torque dos membros inferiores dos joelhos direito e esquerdo, nos movimentos de extensão e flexão.

Tabela 2 - Pico de torque dos joelhos direito e esquerdo nos movimentos de extensão e flexão, nas velocidades angulares de $60 \%$ s e $180 \%$ s.

\begin{tabular}{lcc}
\hline Medidas & Média & $\begin{array}{c}\text { Desvio } \\
\text { padrão }\end{array}$ \\
\hline Extensão 60\%/s esquerdo & 83,1 & 16,8 \\
Extensão 60\%/s direito & 75,0 & 25,7 \\
Flexão 60\%/s esquerdo & 43,2 & 12,9 \\
Flexão 60\%/s direito & 42,8 & 13,0 \\
Extensão 180\%/s esquerdo & 53,4 & 9,2 \\
Extensão 180\%/s direito & 51,9 & 13,8 \\
Flexão 180\%/s esquerdo & 33,0 & 8,9 \\
Flexão 180\%/s direito & 32,9 & 11,0 \\
\hline
\end{tabular}

Foi possível verificar que o pico de torque é maior no joelho esquerdo e no movimento de extensão, na velocidade angular de $60 \%$ s. Já em uma comparação entre o joelho esquerdo e o direito, notou-se que os músculos extensores de joelho são mais fortes que os flexores, o que vem ao encontro do estudo de Potulski e colaboradores (2011). Na velocidade angular de $180 \%$ s, não foram encontradas diferenças significativas entres os membros direito e esquerdo, assim como entre os movimentos de extensão/flexão. Segundo Dvir (2002), a comparação entre grupos flexores bilaterais e extensores bilaterais pode mostrar algum desequilíbrio, contudo este é considerado normal, entre o membro dominante e o não dominante, quando não passa de $10 \%$. 
Em outro estudo, há evidência de que a força dos extensores do joelho, quando preservada, está associada a um melhor desempenho nas atividades da vida diária e até mesmo na prevenção de quedas. (ORDWAY et al., 2006). Conforme Alves et al. (2012), a musculatura extensora do joelho é mais solicitada nas ações diárias por ser maior e ter mais facilidade de recrutamento de fibras musculares.

A avaliação do dinamômetro isocinético para membro superior foi realizado em dez mulheres. Quatro idosas foram excluídas do teste proposto para membro superior por apresentarem alguma patologia que afeta a força muscular ou capacidade funcional, ou, ainda, por outros problemas musculoesqueléticos que comprometem a sua integridade física. Na Tabela 3, apresentam-se os resultados do pico de torque dos ombros, nos movimentos de extensão/flexão bilateralmente, nas velocidades angulares de $60^{\circ} / \mathrm{s}$ e $120^{\circ} / \mathrm{s}$.

Tabela 3 - Pico de torque dos ombros direito e esquerdo, nos movimentos de extensão e flexão, nas velocidades angulares de $60 \%$ s e $120 \%$ s.

\begin{tabular}{lcc}
\hline Medidas & Média & $\begin{array}{c}\text { Desvio } \\
\text { padrão }\end{array}$ \\
\hline Extensão 60\%/s esquerdo & 40,8 & 12,2 \\
Extensão 60\%/s direito & 43,4 & 9,5 \\
Flexão 60\%/s esquerdo & 29,3 & 6,2 \\
Flexão 60\%s direito & 27,9 & 7,0 \\
Extensão 180\%/s esquerdo & 38,7 & 10,7 \\
Extensão 180\%/s direito & 40,5 & 7,0 \\
Flexão 180\%/s esquerdo & 26,6 & 5,4 \\
Flexão 180\%/s direito & 25,8 & 7,2 \\
\hline
\end{tabular}

Dvir (2002), em suas descobertas, e apoiado por outros estudos sobre a ordem do movimento nos músculos do ombro, verificou que os adutores foram mais fortes que os abdutores; os extensores, mais fortes que os flexores; e os flexores, mais fortes que os abdutores, ordem que foi verdadeira para todas as velocidades, para os dois modos de contração (concêntrica/excêntrica) e para as mulheres e os homens de forma semelhante.

De acordo com os resultados da pesquisa, constatou-se que os membros inferiores foram mais influenciados pela prática da atividade física que os membros superiores. Conforme a descrição das características sociodemográficas, 0 índice dos fatores de riscos e morbidades demonstra que essas idosas estão ativas e, assim, sofrem menos os efeitos do envelhecimento.

\section{Conclusões}

No presente estudo, encontrou-se o melhor resultado na avaliação de membro inferior no movimento de extensão, evidenciando que a musculatura do quadríceps está mais forte em relação às demais. No que se refere ao membro superior, sugere-se a realização de mais atividades com pesos. Nesse sentido, ressalta-se a importância de um programa de treinamento de exercícios físicos, no intuito de melhorar a aptidão física e reduzir o número de lesões e quedas entre idosos, prolongando, assim, sua autonomia funcional ao longo da vida. 


\section{Description of the evaluation of muscle strength in aging people who practice physical activities}

\section{Abstract}

As we age many losses happen mainly functional and musculoskeletal. The main objective of this research is to measure bilaterally the muscle peak torque of the upper and lower limbs in the movements of flexion and extension. The study is quantitative descriptive. Fourteen women with the average age of 71.7 were evaluated and all of the participants practiced physical activities in a gym in the city of Passo Fundo RS. The measure of muscular strength was calculated using a dynamometer isokinetic computerized Biodex ${ }^{\mathrm{TM}}$ Multi Joint System 3 Pro $^{\circledR}$. The test was made bilaterally in the movements of extension/flexion of the knee in the angular velocity of $60 \% \mathrm{~s} \mathrm{e}$ $180 \%$ s, and for the shoulder it was used an angular velocity of $60 \%$ s e $120 \%$ s. We can verify that the peak torque is bigger in the left knee in the movement of extension in the angular velocity of $60 \%$ s. In the upper limbs, in the angular velocity of $60 \% \mathrm{~s}$ and $120 \%$ s is bigger in the movement of extension. According to the results of this research, we observed that the lower limbs were more influenced than the upper limbs by the practice of physical activity. Therefore, a program of physical activity is important to improve physical aptitude and to prolong functional autonomy throughout a lifetime.

Keywords: Aging. Muscle strength dynamometer. Knee. Shoulders.

\section{Referências}

ALVES, M. G. S. et al. Força muscular isocinética em idosas: comparação entre o perfil psicológico de gêneros. Revista Brasileira da Ciência e Movimento, São Caetano do Sul, SP, v. 20, n. 4, p. 5-12, 2012.

BARBOSA, A. R. Treinamento contra resistência e função muscular em idosos. Revista Digital, Buenos Aires, ano 12, n. 108, maio 2007. Disponível em: <http://www.efdeportes. com/efd108/treinamento-contra-resistencia-e-funcao-muscular-em-idosos.htm>. Acesso em: 20 mar. 2011.

BIODEX. Biodex TM Multi Joint System 3 Pró. Manual: aplications/operations. American Sports medicine Institute: Birmingham, Alabama, Biodex, 2002. p. 32-35.

DVIR, Z. Isocinética: avaliações musculares, interpretações e aplicações clínicas. Barueri, SP: Manole, 2002.

ERNESTRO, C. et al. Efeitos de diferentes intervalos de recuperação no desempenho muscular isocinético em idosos. Revista Brasileira de Fisioterapia, São Carlos, ano 13, n. 1, p. 65-72, jan./fev. 2009.

ORDWAY, N. R. et al. Reliability of knee and ankle strength measures in an older adult population. Journal of Strength \& Conditioning Research, Colorado Springs, v. 20, n. 1, p. 82-87, feb. 2006.

POTULSKI, A. P. et al. Pico de torque muscular de flexores e extensores de joelho de uma população geriátrica. Revista Brasileira de Ciências da Saúde, São Caetano do Sul, ano 9, n. 28, p. 25-30, abr./jun. 2011.

PRENTICE, W. E.; VOIGHT, M. L. Técnicas em reabilitação musculoesquelética. Porto Alegre: Artmed, 2007.

ULBRICHT; V. R.; CASSOL; M. P. Adaptando tecnologia da informação e comunicação ao estilo do idoso para proporcionar um maior conhecimento através de sua representação cognitiva. In: CONGRESSO INTER- 
NACIONAL DE EDUCAÇÃO A DISTÂNCIA, 12, Florianópolis, 2005. Disponível em: <http://www.abed.org.br/congresso2005/por/ pdf/203tcc5.pdf>. Acesso em: 23 maio 2011.

SONZA, A.; ANDRADE, M. C. Análise das curvas de torque isocinético em movimentos de ombro. Revista Brasileira de Medicina do Esporte, São Paulo, v. 18, n. 12, p. 91-94, mar./abr. 2012.

TARTARUGA, M. P. et al. Treinamento de força para idosos: uma perspectiva de trabalho multidisciplinar. Revista Digital, Bueno Aires, ano 10, n. 82, mar. 2005. Disponível em: <http://www.efdeportes.com/efd82/treinam.htm>. Acesso em: 20 mar. 2011.

WORLD HEALTH ORGANIZATION (WHO). Obesity: preventing and managing the global epidemic. Report of a WHO consultation on obesity. Geneva: World Health Organization, 1997. p. 107-158. 